\title{
Hairy Cell Leukemia: A Clinicopathological Study of 18 Cases
}

\author{
Kazoomi Patel', Rupal shah ${ }^{1 *}$, Biren Parikh ${ }^{2}$, Jyoti Sawhney ${ }^{2}$ and Bina Brahmbhatt ${ }^{2}$ \\ 'Pathology Dept, GCS medical college, hospital and Research centre,Naroda road, Ahmedabad, Gujarat. India \\ ${ }^{2}$ Pathology dept,Gujarat Cancer \& Research Institute, Asarva, Ahmedabad, Gujarat, India
}

\begin{abstract}
Introduction: Hairy cell leukemia (HCL) is an uncommon chronic B-cell lymphoproliferative disorder. It affects primarily elderly men and presents with splenomegaly, pancytopenia and monocytopenia. It has to be differentiated from various chronic lymphoproliferative disorders (CLPDs) because of different protocol of treatment and clinical course.

Methods: This study included 18 cases of HCL out of 300casesof CLPDs diagnosed over a period of 4 years (2009-2012) at tertiary cancer centre. All 18 cases were evaluated for clinical history, signs and symptoms, laboratory data, all initial and follow up peripheral blood smear (PBS) examinations, bone marrow aspirate (BMA) with imprint smear, bone marrow biopsy, immunophenotyping data, treatment details and response to treatment from medical records.

Results: This study included 18 cases, age group of 35-69 years, with a median age of 40years and male predominance. M:F ratio was $6: 1$. HCL was $6 \%$ out of allCLPDs. Patients were presented with common complaints of weakness, fever and abdominal pain. On clinical examination, commonest findings were pallor, splenomegaly, hepatomegaly and abdominal lump. On laboratory investigations, 17 cases had anemia, 4 cases had leucocytosis, 11 cases had leucopenia and 11 cases had pancytopenia. On bone marrow biopsy,findings were diffuse infiltration of the marrow by atypical lymphoid cells having abundant cytoplasm. For immunophenotyping all patients` blood samples were CD19 gated on lymphocytes. It showed co expression of CD103, CD11c and CD25. Thirteen patients were treated with cladribine based chemotherapy with excellent responses.
\end{abstract}

Conclusion: HCL clinically presents with pancytopenia and splenomegaly in middle aged male but few patient can present with unusual clinical features like absence of a palpable spleen, generalized lymphadenopathy and leucocytosis.

\section{Keywords: Hairy Cell Leukemia, Flow Cytometry, Cladribine, Bone Marrow Aspiration}

\section{Introduction}

Hairy cell leukemia (HCL) is an uncommon but distinct form of chronic B- cell lymphoproliferative disorder comprising about $2 \%$ of lymphoid leukemias. It affects primarily elderly men and is characterized by triad of splenomegaly, pancytopenia and monocytopenia. ${ }^{[1]}$ Most of patients with HCL present with involvement of bone marrow, spleen and few cases with peripheral blood. Hairy cell have characteristic appearance of small to medium in size, moderate nuclear: cytoplasmic ratio, round to oval nuclei and having abundant cytoplasm exhibiting thin circumferentially cytoplasmic projections.(figure 1)

The differential diagnosis of HCL from other chronic B-cell lymphoproliferative disorders is clinically important because patients with HCL respond better and highly sensitive to cladribine (2-chlorodeoxyadenosine ) a purine analogues and pentostatine (2- deoxycoformycin) based chemotherapy, but do not respond well to conventional lymphoma chemotherapy. These drugs give long term complete remission even in a significant portion of HCL patients with relapsed disease. ${ }^{[2-5]}$

The final diagnosis of HCL mainly based on morphologic findings of hairy cells on peripheral blood smear(PBS), bone marrow(BM) and Flow cytometric immunophenotyping (IPT) of malignant cells. HCL has a characteristic immunophenotypic profile and light scatter characteristics. The tumor cells express B cell- associated markers CD19, CD20, CD22 and CD79b with characteristically positive for CD103, CD25, CD11c and usually negative for CD10, CD5 and CD23 Coexpression of CD103, CD25 and CD11c is unique for HCL, It is an absolute criteria for diagnosis of HCL . However atypical IPT have been reported in morphologically classical HCL. ${ }^{[6]}$ In this study we evaluate clinicopathological features, immunophenotypic profile and treatment details.

\section{Materials and Methods}

This retrospective study included 18 cases out of 300 cases of CLPDs diagnosed over a period of 4 years ( January 
2009 to December 2012) at tertiary cancer centre. HCL cases were suspected morphologically and diagnosis was confirmed by immunophenotyping. Total 18 cases of HCL and 1 case of HCL Variant were retrieved from medical records. All 18 cases were evaluated for clinical history, signs and symptoms, laboratory data, all initial and follow up peripheral blood smear (PBS) examinations, bone marrow aspirate (BMA) with imprint smear, bone marrow biopsy, immunophenotyping data, treatment details and response to treatment from medical records.

Morphology: The peripheral smear and bone marrow aspirate smears were stained with wright stain for morphological examination. Bone marrow biopsy or tissue biopsy were stained with Hematoxylin and Eosin (H \& E) stain. (Figure 1)

Immunophenotyping by Flow Cytometry: For flowcytometry (FCM), Ethylene diamine tetra acetic acid (EDTA) sample of BMA/PBS was processed for IPT. The cells were prepared by wash and lyse technique, then mixed with antibody tagged with different fluorochromes. BD FACS CANTO II, a six color FCM was performed. Panel of directly conjugated monoclonal antibodies comprised of CD45 (PerCP), CD3 (PE Cy7), CD5 (PerCP), CD10 (APC), CD19 (APC-H7), CD20 (PE Cy7), CD22 (FITC), CD23 (PE), CD38 (PE), CD56 (APC), CD79b (PE), FMC7 (FITC), Kappa (PE), Lambda (FITC), CD103 (PEA), CD25 (APC-A), CD11c (PE-A) and sIgM (FITC). Minimum 10,000 events were acquired using low side scatter versus low to high forward scatter gating. Data (collected in list mode) were analyzed with Cell Quest pro software (Becton Dickinson) as shown in figure 2.

\section{Results}

Age and sex distribution: Age range was from 35 to 69 years, with median age 40 years. Fifteen out of eighteen patients were male (83\%), M:F ratio was 6:1. Duration of symptoms were from 6 months to 2 years.

Symptoms: Patients most commonly presented with symptoms of weakness (66\%), fever $(50 \%)$, bleeding (33\%), weight loss $(22 \%)$, headache $(11 \%)$ and abdominal pain (11\%). (Table-1)

Physical Examination: Pallor and splenomegaly (extend 2-18 cm below costal margin) were most common physical findings followed by generalized lymphadenopathy and hepatomegaly (extend $2-5 \mathrm{~cm}$ below costal margin). (Table-1)

Laboratory Data: On investigations, (Table-1) 94\% patients had anemia $(\mathrm{Hb}<11 \mathrm{~g} / \mathrm{L})$ with hemoglobin values ranging from $5 \mathrm{~g} / \mathrm{L}$ to $12.2 \mathrm{~g} / \mathrm{L}$. Total leukocyte counts(TLC) ranging from $800 /$ cumm to $2,44,000 /$ cumm with $61 \%$ of the patients (11/18) being leukopenic, while four $(22 \%)$ patients had leukocytosis. Absolute monocyte count was low in $94 \%$ of cases (17/18) with one case of monocytosis. Platelet counts ranged from $35,000 /$ cumm to $2,00,000 /$ cumm with $61 \%(11 / 18)$ patients being thrombocytopenic. Pancytopenia was seen in $11 / 18(61 \%)$ cases. Bone marrow aspiration done in all cases. Bone marrow biopsy was available in 11 cases. We did not performed Tartrate resistant acid phosphatase (TRAP) at this centre.

Immunphenotypic Characteristics: Results of IPT by FCM along with intensity of antigen expression are summarized in (Table - 2). All the cases expressed characteristic HCL phenotype [Figure 2] CD19+, CD20+, $\mathrm{CD} 22+$ and characteristic co-expression markers CD11c+, CD25+ and CD103+. Eight cases (44\%) demonstrated kappa light chain and ten cases (56\%) showed lambda light chain restriction. Two cases (11\%) expressed CD10 and CD23 expression. Only one case showed CD 5 expression.

Treatment and follow up: There were 18 cases of HCL diagnosed. Out of which, 13 cases were treated at same cancer centre. Nine patients received one cycle of cladribine based chemotherapy and all of them achieved complete remission . One patient was treated by splenectomy with supportive care but relapsed after 5 months of initial treatment detected on BMA during follow up, later treated by further 8 cycles of CHOP (cyclophosphamide, Doxorubicin, Vincristine, Prednisolone) chemotherapy. Three patients were poor not affording cladribine based chemotherapy and treated with CHOP based chemotherapy. One out of these three patients relapsed at 7 months after initial treatment and expired due to complications of neutropenia and its infective complications. All patients received neutropenia care. Five Patients were not appeared for treatment. Detailed follow up of treated patients was available for period of two months to sixty months after initial treatment. All these patients were alive and disease free without any signs, symptoms, organomegaly and had complete blood count within normal range.

\section{Discussion}

Accurate diagnosis of HCL is critical because therapy with purine analogues cladribine associated with high complete response rate and long relapse-free survival in patients with HCL but is less effective in patients with other chronic B-cell leukemias or lymphomas. ${ }^{[2-7]}$ The diagnosis of HCL is usually made by examining the morphologic features of the hairy cell on PBS and BMA in conjunction with the characteristic immunophenotyping by FCM analysis. HCL constitutes approximately $6 \%$ of all CLPDs presenting at this institute. ${ }^{[6]}$ This figure is much higher than incidence 
TABLE 1: Clinical presentation with laboratory data in HCL cases.

\begin{tabular}{|c|c|c|c|}
\hline Variables & Manifestations & Number Of Cases $\mathrm{N}=18$ & Percentage $(n=18, \%)$ \\
\hline \multirow{7}{*}{ Symptoms } & Weakness & 12 & 66 \\
\hline & Fever & 09 & 50 \\
\hline & Bleeding & 06 & 33 \\
\hline & Weight loss & 04 & 22 \\
\hline & Headache & 02 & 11 \\
\hline & Abdominal pain & 02 & 11 \\
\hline & Lump in abdomen & 02 & 11 \\
\hline \multirow{4}{*}{ Signs } & Pallor & 15 & 83 \\
\hline & Splenomegaly & 14 & 78 \\
\hline & Hepatomegaly & 04 & 22 \\
\hline & Lymphadenopathy & 07 & 38 \\
\hline \multirow{6}{*}{ Lab data } & Anemia & 17 & 94 \\
\hline & Thrombocytopenia & 11 & 61 \\
\hline & Leucopenia & 11 & 61 \\
\hline & Leucocytosis & 04 & 22 \\
\hline & Pancytopenia & 11 & 61 \\
\hline & Monocytosis & 01 & 5 \\
\hline
\end{tabular}

Table 2: Results of Flow Cytometric Immunophenotyping in HCL.

\begin{tabular}{|l|c|c|}
\hline Surface Marker & No. of positive cases (\%) $\mathbf{N}=18$ & Intensity \\
\hline CD19 & $18(100)$ & +++ \\
\hline CD20 & $18(100)$ & +++ \\
\hline CD22 & $18(100)$ & +++ \\
\hline CD79b & $11(61)$ & ++ \\
\hline FMC 7 & $16(88)$ & +++ \\
\hline Light chain restriction & Kappa 08 (44) \\
\hline LD5 & $01(5)$ & ++ \\
\hline CD10 & $02(11)$ & ++ \\
\hline CD23 & $02(11)$ & ++ \\
\hline CD11c & $18(100)$ & ++ \\
\hline CD25 & $18(100)$ & ++ \\
\hline CD103 & $18(100)$ & ++ \\
\hline
\end{tabular}

Table 3: Clinical presentation and laboratory characteristics in various studies.

\begin{tabular}{|c|c|c|c|c|}
\hline $\begin{array}{l}\text { Feature at } \\
\text { presentation }\end{array}$ & Bouroncle et al $(n=82)^{(7)}$ & Chatterjee et al $(n=15)^{(8)}$ & Galani et al $(n=28)^{(9)}$ & Present study $(n=18)$ \\
\hline $\begin{array}{l}\text { Age of youngest pt at } \\
\text { diagnosis }\end{array}$ & 22 yrs & 32 yrs & 26 yrs & 35 yrs \\
\hline$M: F$ & $4.2: 1$ & $2: 1$ & $6: 1$ & $6: 1$ \\
\hline Pancytopenia & $50 \%$ & $60 \%$ & $54 \%$ & $61 \%$ \\
\hline Anaemia (<110gm/L) & $84 \%$ & $67 \%$ & $88 \%$ & $94 \%$ \\
\hline $\begin{array}{l}\text { Thrombocytopenia } \\
\text { (<1lakh/cmm) }\end{array}$ & $58 \%$ & $60 \%$ & $77 \%$ & $55 \%$ \\
\hline $\begin{array}{l}\text { Leucocytogsis } \\
(>11.0 \times 10 / L)\end{array}$ & - & $1 \%$ & $8 \%$ & $22 \%$ \\
\hline $\begin{array}{l}\text { Absence of palpable } \\
\text { spleen }\end{array}$ & $7 \%$ & 0 & $4 \%$ & $22 \%$ \\
\hline Hepatomegaly & $58 \%$ & $53 \%$ & $28 \%$ & $22 \%$ \\
\hline Lymphadenopathy & $23 \%$ & $13 \%$ & $24 \%$ & $38 \%$ \\
\hline
\end{tabular}




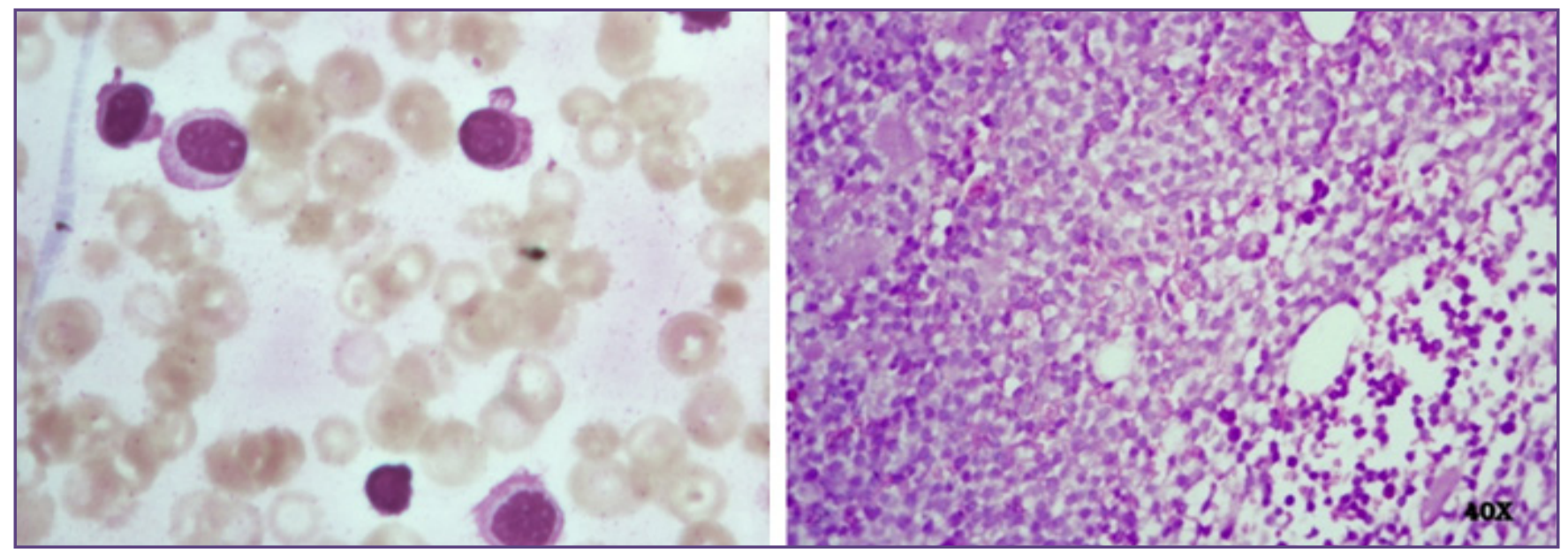

Fig. 1; Peripheral blood smear of classical hairy cells with cytoplasmic projections( Wright stain,x1000) and Bone marrow biopsy of hairy cell leukemia (H and E stain,x400).
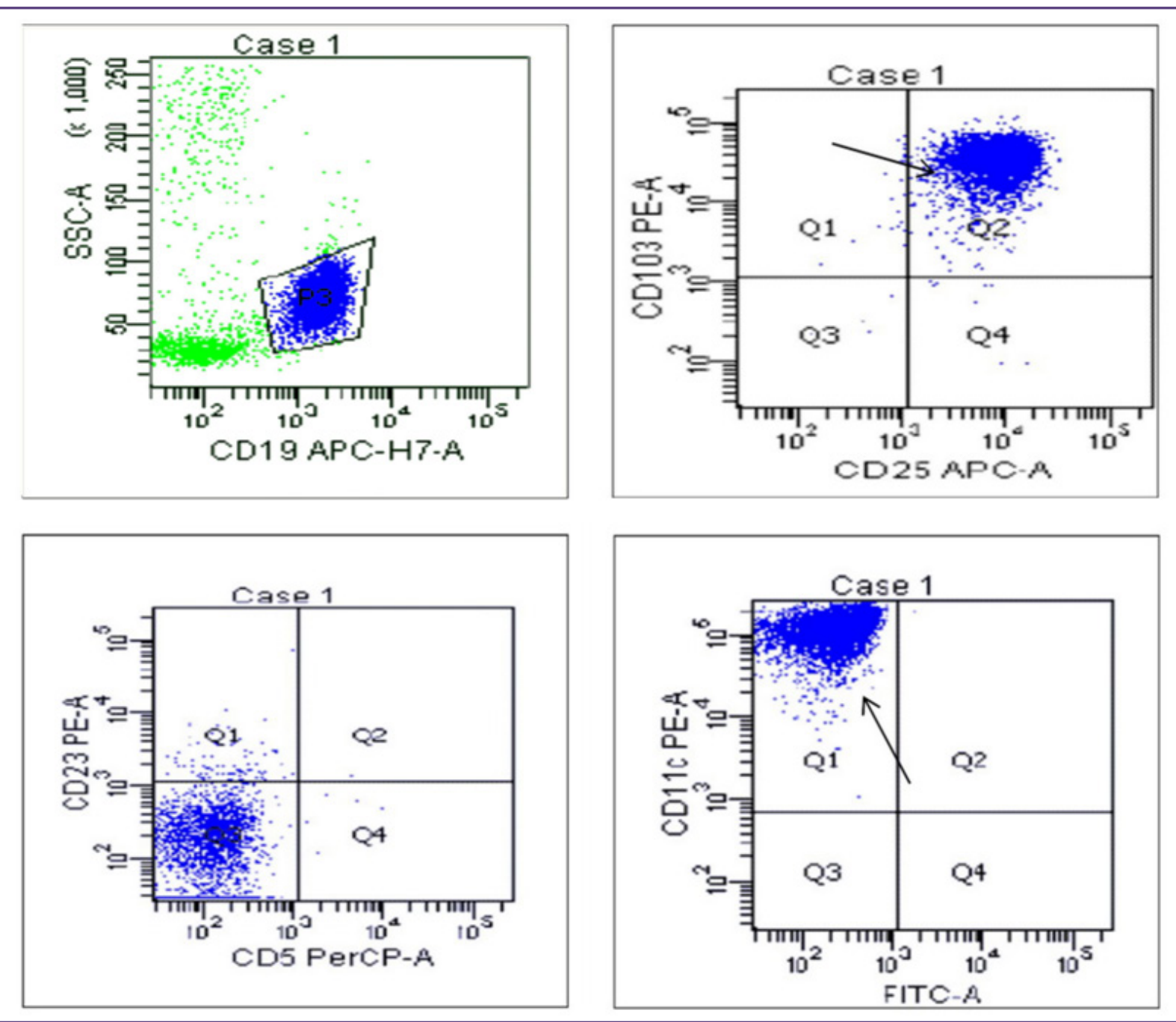

Fig. 2: Flow cytometry dot plots of CD19 gated cells of HCL. Hairy cells express CD25, CD103 and CD11c. 
of $2 \%$ reported in western literature. ${ }^{[1]} \mathrm{HCL}$ patients are symptomatic and referred to higher tertiary cancer centers for early management by physicians. This could explain higher incidence in this study compared to Western. In this study of 18 cases, median age being 40 years (ranging from 35 to 69 years), which is quite younger; however, it is correlated with the findings of another Indian study by Chatterjee $\mathrm{T}$ et al. ${ }^{[8]}$ The youngest patient in this study was 35 years old comparable to that reported by Chatterjee $\mathrm{T}$ et al. ${ }^{[8]}$ was 32 years. As per WHO, male predominance was also seen in this study $(\mathrm{M}: \mathrm{F}=6: 1)$. All the patients presented with symptoms like easy fatigability, fever, bleeding manifestations and weakness, all of which were reported in standard references. ${ }^{[1,7,8,9,10,11]}$ On physical examination, splenomegaly was present in $78 \%$ of cases, which was less than the other studies. ${ }^{[7-9]}$ Hepatomegaly was seen in $22 \%$ cases similar to findings of Galani et al. ${ }^{\left[{ }^{[9}\right.}$ Though uncommon, lymphadenopathy was seen in $7 / 18(38 \%)$ cases, all patients had generalized lymphadenopathy which is higher than values reported by other studies. ${ }^{[7,9]}$ Anemia was the most common hematologic abnormality (94\%) similar to other study. ${ }^{[9]}$ Four patients had leukocytosis. Cases of HCL with profound lymphocytosis have been reported only rarely in the literature. ${ }^{[12]}$ These findings show most patients with classic HCL usually present with pancytopenia, but rare cases can present with leukocytosis. This possibility should be kept in mind to provide accurate diagnosis and proper treatment.

Accurate diagnosis of HCL is critical because therapy with purine analogues is associated with high complete response rates and long relapse-free survival in patients with HCL but is less effective in patients with other CLPDs. ${ }^{\left[{ }^{9]}\right.} \mathrm{HCL}$ is a monoclonal B-cell neoplasm with coexpression of CD19, CD103, CD11c and CD25. In the present study, 11\% of the patients analyzed were CD10 positive which is within range of $5 \%$ to $26 \%$ reported by various studies. ${ }^{[6,9,13]}$ In the present study, CD23 positivity was seen in $11 \%$ of HCL compared to $19 \%$ of study by Galani KS et al. ${ }^{\left[{ }^{[9]}\right.}$ One patient in the present study had a transformation to large cell non hodgkin's lymphoma.

The value of FCM analysis of peripheral blood for diagnosis of HCL appears to be under-recognized by many physicians, perhaps due to a lack of familiarity with the capabilities of this technique and a misperception that leukopenic patients have very few circulating hairy cells for detection. ${ }^{[9]}$ FCM is ideally suited for the diagnosis and therapeutic monitoring of diseases like HCL with low numbers of malignant cells in peripheral blood.
Limitations: The diagnosis of HCL cases was based on a specific fixed panel of antibodies. TRAP was not available at this centre. Some patients didn't appear for treatment.

\section{Conclusions}

Higher incidence of HCL ( $6 \%$ of all CLPDs) at this centre may be due to referral tertiary cancer center. HCL can occur at a younger age, which may be due to geographical variation. Unusual clinical presentations of patients include absence of palpable spleen, presence of lymphadenopathy and leukocytosis. Variable expression of CD10 and CD23 can also be seen in HCL which are commonly used for diagnosis of follicular lymphoma and chronic lymphocytic leukemia. For accurate diagnosis of HCL, clinic-pathological correlation is needed. Excellent response to cladribine based chemotherapy is noted in majority of the patients.

\section{Abbreviations}

HCL- Hairy cell leukemia, BMA- bone marrow aspiration, BMB- bone marrow biopsy, PBS- peripheral blood smear, IPT- immunophenotyping, CLPD- chronic lymphoproliferative disorders, FCM- flowcytometry, CDclusters of differentiation

\section{References}

1. Jaffe ES, Harris NL, Stein H, et al. editors. Pathology and Genetics of Tumours of Haematopoietic and Lymphoid Tissues. World Health Organization Classification of Tumors. 4th ed. Lyon, France: IARC Press; 2008.P.188-90.

2. Jehn U, Bartl R, Dietzfelbinger H, et al. An update: 12-year follow-up of patients with hairy cell leukemia following treatment with 2-chlorodeoxyadenosine. Leukemia. 2004; 18:1476-1481.

3. Robak T, Blasinska-Morawiec M, Blonski J, et al. 2-chlorodeoxyadenosine (cladribine) in the treatment of hairy cell leukemia and hairy cell leukemia variant: 7-year experience in Poland. Eur J Haematol.1999; 62:49-56.

4. Finn IW, Kopecky KJ, Foucar MK, et al. Long term follow up of remission duration, mortality and second malignancies in hairy cell leukemia patients treated with pentostatin. Blood . 2000; 96(9):2981-2986.

5. Tallman MS. Current treatment strategies for patients with hairy cell leukemia. Rev clin Exp Hematol. 2002; 6:389-400.

6. Jasionowski TM, Hartung L, Greenwood JH, et al. Analysis of CD10+ hairy cell leukemia. Am J Clin Pathol.2003; 120(2):228-235.

7. Bouroncle B. Leukemic reticuloendotheliosis (hairy cell leukemia). Blood. 1979; 53:412-36.

8. Chatterjee T, Panigrahi I, MahapatraM, Pati HP, Kumar R, Naithani R, et al. Hairy cell leukemia: Clinical, pathological and ultrastructural findings in Asian-Indians. Indian J Cancer. 2008; 45(2):41-44. 
9. Galani KS, Subramanian PG, Gadage VS, Rahman K, Ashok Kumar MS, Shinde S, et al. Clinico-pathologicalprofile of Hairy cell leukemia: Critical insights gained at a tertiary care cancer hospital. Indian J Pathol Microbiol. 2012; 55(1):61-65.

10. Malfuson JV, Gisserot O, Souleau B et al. Hairy Cell Leukemia: 30 cases and review of the literature. Ann Med Interne (Paris). 2003; 154:435-40.

11. Hoffman MA. Clinical presentations and complications of hairy cell leukemia. Hematol Oncol Clin North Am. 2006; 20(5):1065-73

12. Adley BP, Sun X, Shaw JM, Variakojis D. Hairy Cell Leukemia with Marked lymphocytosis. Arch Pathol Lab Med. 2003; 127(2):253-54.

13. Bacal NS. Flow cytometry: Immunophenotyping in 48 hairy cell leukemia cases and the relevance of fluorescence intensity in CDs expression for diagnosis. Einstein. 2007; 5(2):123-128.

*Corresponding author:

Dr. Rupal Shah., 401, Ananya Residency, Near Mayur School, Paliyadnagar, Naranpura, Ahmedabad, Gujarat. Pin 380013, India

Phone: +919825586560

Email: rupu_desai@yahoo.co.in

Financial or other Competing Interests: None. 